\title{
Synthesis and Optical Properties of CuO Nanocrystals with Controllable Shapes and Size
}

\author{
Neelinder Goraya ${ }^{1}$, Satbir Singh ${ }^{2}$ \\ ${ }^{1,2}$ Department of Electronics and Communication, GNDU-Regional Campus, Gurdaspur, Punjab, India
}

\begin{abstract}
Highly dispersed copper oxide $(\mathrm{CuO})$ nanoparticles had been successfully prepared by a hydrothermal technique where different adding temperatures of $\mathrm{NaOH}$ were taken. The as-prepared $\mathrm{CuO}$ nanoparticles were characterized by X-ray diffraction (XRD), transmission electron microscopy (TEM) and Raman spectroscopy. The results showed that the adding temperature of $\mathrm{NaOH}$ has an important influence on the shape and size of $\mathrm{CuO}$ nanocrystals The influence of reaction conditions on morphology of $\mathrm{CuO}$ nanocrystals was discussed. Furthermore, different shapes of obtained $\mathrm{CuO}$ nanocrystals exhibit different Raman properties.
\end{abstract}

\section{Introduction}

Nano- structured transition metal oxides (MOs) are the indisputable prerequisite for the development of various novel functional and smart materials. These transition MO nanocrystals have been attracting much attention not only for fundamental scientific research, but also for various practical applications because of their unique physical and chemical properties [1]. As an important ptype semiconductor metal oxide with a narrow band gap $(1.2 \mathrm{eV})$, copper oxide $(\mathrm{CuO})$ has been extensively studied because of its diverse applications as material for heterogeneous catalysts, gas sensors, optical switch, magnetic storage media, field emission devices, solar cells, etc[2]. $\mathrm{CuO}$ nanostructures are also considered as electrode materials for the next-generation rechargeable lithium-ion batteries (LIBs) because of their high theoretical capacity, safety, and environmental friendliness. They are also promising materials for the fabrication of solar cells because of their high solar absorbance, low thermal emittance, relatively good electrical properties, and high carrier concentration.

Research on nanoparticles has gathered wide attention during the last decade because of their unusual and size dependent optical, magnetic, electronic, and chemical properties. To fully utilize these properties, the size and shape must be well controlled. Some synthesis methods are successful for fabricating shape-controlled nanocrystals of metals, semiconductors and complex metal oxides [3]. In recent years, the shape controlled synthesis of nanostructured $\mathrm{CuO}$ has attracted considerable attention. However, the exciting techniques have to rely on the proper selection of expensive surfactants or high crystallization temperatures. Therefore, seeking a simple strategy for low-cost, low- temperature, controlled synthesis of oxide nanostructures at mild conditions is highly desired, and it is important as well for exploring zero- and one-dimensional oxide-based nanostructures for applications in nanodevices [4]. Here, we describe a simple procedure for the synthesis of shape-controlled $\mathrm{CuO}$ nanocrystals. Here we use hydrothermal synthesis of $\mathrm{CuO}$ nanoparticles which is generally based on a two-step process. First, cupric hydroxide $\left[\mathrm{Cu}(\mathrm{OH})_{2}\right]$ particles are formed by the reaction of a cupric salt precursor with a basic solution, such as sodium hydroxide $(\mathrm{NaOH})$ or ammonium hydroxide. The $\mathrm{Cu}(\mathrm{OH})_{2}$ particles are then thermally dehydrated in an autoclave at fixed temperatures to obtain the final $\mathrm{CuO}$ nanoparticles. With the hydrothermal techniques different sized $\mathrm{CuO}$ nanocrystals can be obtained by modulating the adding temperatures of $\mathrm{NaOH}$. The method provides a convenient, low-cost, nontoxic route for the synthesis of nanostructures of oxide materials.

\section{Experimental}

\subsection{Sample Preparation}

Analytically pure $\mathrm{Cu}\left(\mathrm{NO}_{3}\right)_{2}$ and $\mathrm{NaOH}$ were used as the starting materials without further purification. In a typical procedure, two identical sets of $300 \mathrm{~mL}$ of $0.02 \mathrm{M}$ $\mathrm{Cu}\left(\mathrm{NO}_{3}\right)_{2}$ solution were prepared by dissolving $\mathrm{Cu}\left(\mathrm{NO}_{3}\right)_{2} \cdot 3 \mathrm{H} 2 \mathrm{O}$ in deionized water. Each set of solution was added into a round-bottom flask equipped with a refluxing device. The two sets of $\mathrm{Cu}\left(\mathrm{NO}_{3}\right)_{2}$ solution were kept at an appropriate temperature $\left(60^{\circ} \mathrm{C}\right.$ and $100^{\circ} \mathrm{C}$, respectively) with vigorous stirring; then $0.50 \mathrm{~g}$ of solid $\mathrm{NaOH}$ (platelets) was rapidly added into each set of solution, where a large amount of black precipitate was

\section{${ }^{1}$ Neelinder Goraya: gneelinder@yahoo.com}


simultaneously produced and maintained at the crystallization temperature for $10 \mathrm{~min}$. After all reactions were completed, the resulting products were centrifuged, washed with water and ethanol for several times, and dried in air at room temperature.

\subsection{Characterization}

Crystal structure identification was made by X-ray diffraction (XRD) using a Bruker D8 Advance X-ray diffractometer with $\mathrm{Cu} \mathrm{K} \quad(\lambda=0.15405 \mathrm{~nm})$ radiation. Transmission electron microscopy (TEM) measurements were performed on a JEOL JEM-2100 microscope operating at $200 \mathrm{kV}$, by depositing a drop of $\mathrm{CuO}$ dispersion onto 300 mesh $\mathrm{Cu}$ grids coated with carbon layer. Laser Raman Spectroscopy (LRS) studies were carried out using a Renishaw Invia Raman spectrometer from 100 to $700 \mathrm{~cm}^{-1}$ at room temperature.

\section{Results and discussion}

XRD pattern (Fig. 1) of the products obtained is identical to the single-phase $\mathrm{CuO}$ with a monoclinic structure and the diffraction data are in good agreement with JCPDS card of CuO (JCPDS 80-1268). No peaks of impurity are observed in the XRD pattern. The broadening of the peaks indicates the small size of the products [5]. Actually, the average sizes of the $\mathrm{CuO}$ nanocrystals prepared at adding temperatures of $60^{\circ} \mathrm{C}$ and $100^{\circ} \mathrm{C}$, are estimated to be 15 and $16 \mathrm{~nm}$, respectively, according to the Scherrer equation. It was found that the adding temperature of $\mathrm{NaOH}$ has an important influence on the shape and size of $\mathrm{CuO}$ nanocrystals.

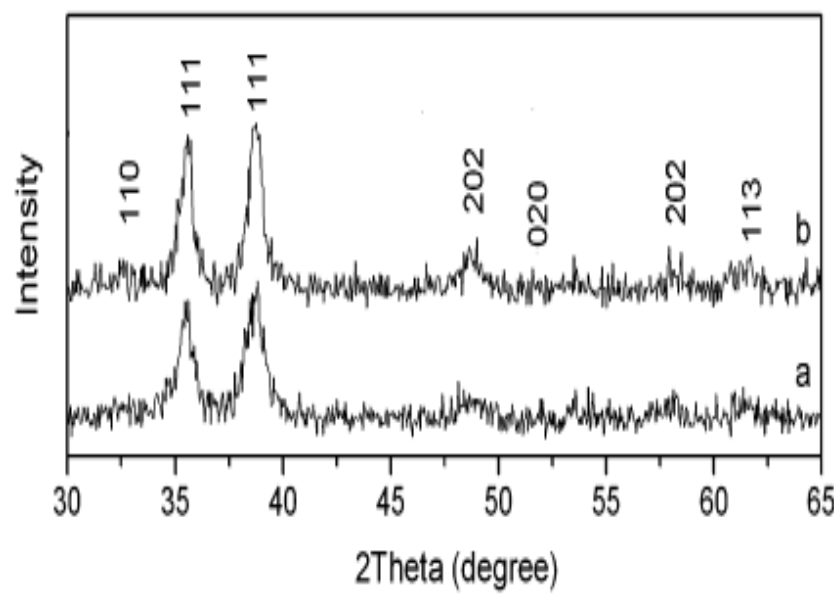

Figure 1. XRD patterns of $\mathrm{CuO}$ products prepared at different adding temperatures of $\mathrm{NaOH}$ : (a) $100{ }^{\circ} \mathrm{C}$ [4]; (b) $60{ }^{\circ} \mathrm{C}$ [4]

As can be seen from Fig. 2a, the nanorods with diameters of $5 \mathrm{~nm}$ and lengths of 25-40 nm were formed when $\mathrm{NaOH}$ was added at $100^{\circ} \mathrm{C}$. When the adding temperature decreased to $60^{\circ} \mathrm{C}$, the $\mathrm{CuO}$ nanobelts with diameters of 8-40 $\mathrm{nm}$ and lengths up to $1.0 \mu \mathrm{m}$ were obtained (Fig. $2 b)$.

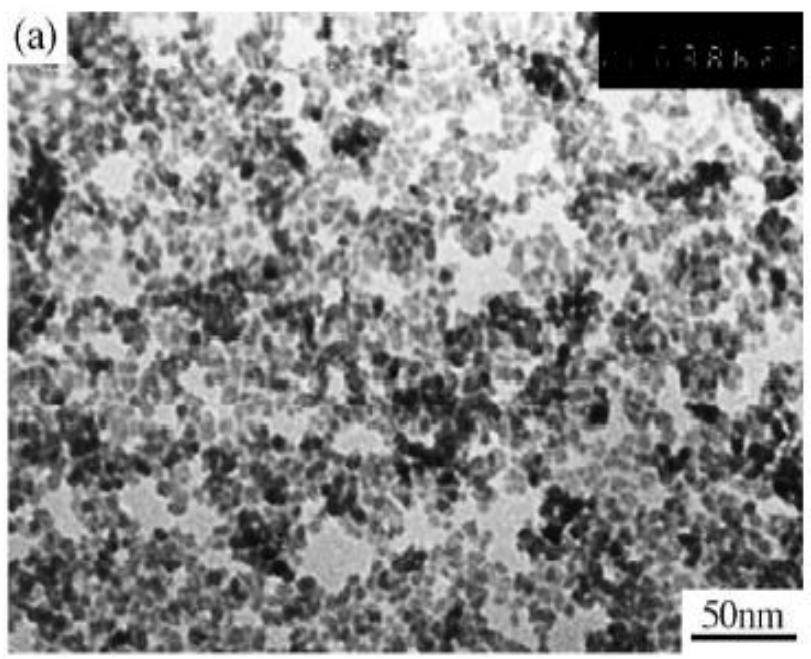

(a)

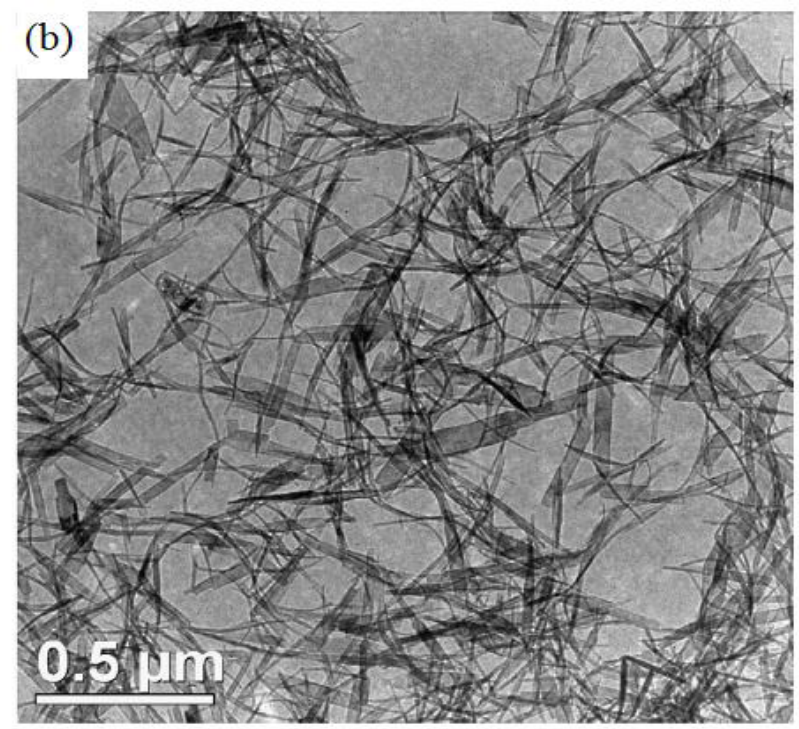

(b)

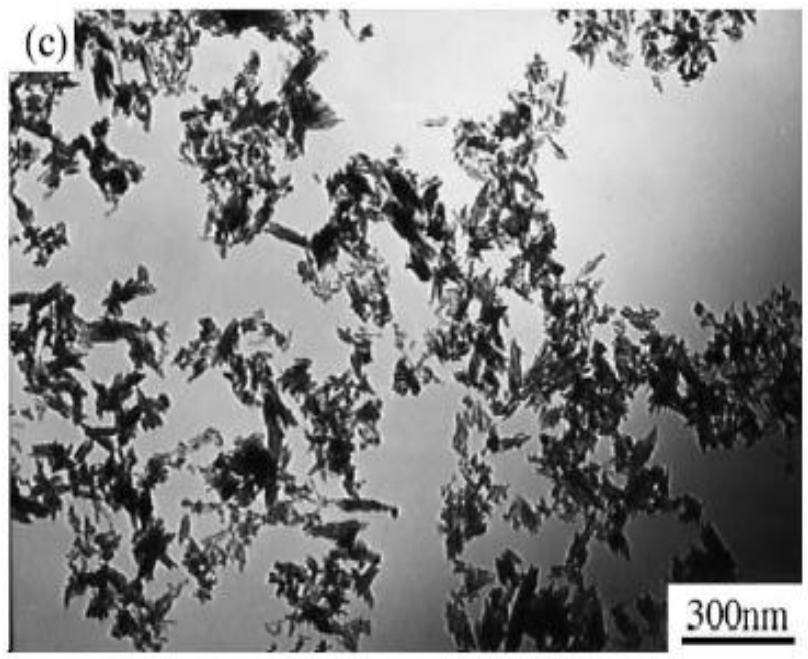

(c) 


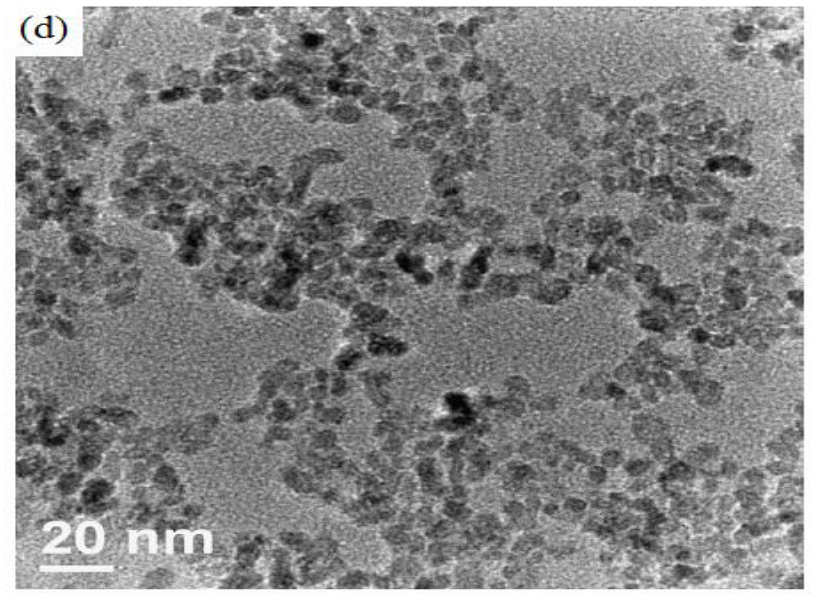

(d)

Figure 2. TEM images of the as-prepared $\mathrm{CuO}$ nanoparticles: (a) adding $\mathrm{NaOH}$ at $100^{\circ} \mathrm{C}$ [4]; (b) adding $\mathrm{NaOH}$ at $60^{\circ} \mathrm{C}$ [4]; (c) in the absence of glacial acetic acid and $\mathrm{NaOH}$ [6]; (d) in the presence of glacial acetic acid and $\mathrm{NaOH}$ at $100^{\circ} \mathrm{C}$ [4].

Typical TEM image of the as-prepared $\mathrm{CuO}$ nanoparticles is presented in Fig. 2, showing that the products are composed of spherical particles with narrow size distribution and high dispersion. The size of the particles observed in the TEM image is in the range of 5$7 \mathrm{~nm}$, which is in good agreement with that estimated by Scherrer equation from the XRD pattern.

It is also important to point out that adding a small quantity of glacial acetic acid has an important influence on the shapes of $\mathrm{CuO}$ crystals. As a matter of fact, the $\mathrm{CuO}$ sample had been prepared by adding solid $\mathrm{NaOH}$ into $\mathrm{Cu}\left(\mathrm{NO}_{3}\right)_{2}$ solution at $100^{\circ} \mathrm{C}$ in the presence of $1 \mathrm{~mL}$ glacial acetic acid. The TEM analysis shows that the obtained black precipitate consists of $8 \mathrm{~nm}$ sized spherical particles. In this method, the additive glacial acetic acid was used to prevent the hydrolysis of the copper acetate solution. If glacial acetic acid was not added, the copper acetate solution would directly hydrolyze above $80^{\circ} \mathrm{C}$ in the absence of $\mathrm{NaOH}$ and form well-dispersed needle-shaped $\mathrm{CuO}$ nanocrystals as observed in the TEM image (Fig. 2c). The result also indicates that different morphologies of $\mathrm{CuO}$ nanocrystals can be obtained easily by controlling the addition of glacial acetic acid and $\mathrm{NaOH}$.

Raman spectroscopy is a very useful tool for the study of phases and structures of oxide systems. The Raman frequency can shift, and bandwidth can broaden, with the decreasing microcrystal size [7-8]. Raman spectra of $\mathrm{CuO}$ products prepared at different adding temperatures of $\mathrm{NaOH}$ are illustrated in Fig. 3. For the monoclinic $\mathrm{CuO}$, this belongs to the $C^{6}{ }_{2 h}$ space group with two molecules per unit cell. There are nine zone-center optical phonon modes with symmetries $4 \mathrm{Au}+5 \mathrm{Bu}+\mathrm{Ag}+2 \mathrm{Bg}$. Only three $\mathrm{Ag}+2 \mathrm{Bg}$ modes are Raman active [9-10]. For the sample a (Fig. 3), it can be seen that there are three Raman peaks at 283,330 and $619 \mathrm{~cm}^{-1}$, and these peaks can be assigned to one $\mathrm{Ag}$ and two $\mathrm{Bg}$ modes, respectively. And the Raman peaks of sample b (Fig. 3) have a blue shift and shift to 290,337 and $623 \mathrm{~cm}^{-1}$, respectively. It is clear that, in this case, the reaction temperature plays an important role in the formation of highly dispersed $\mathrm{CuO}$ nanoparticles.

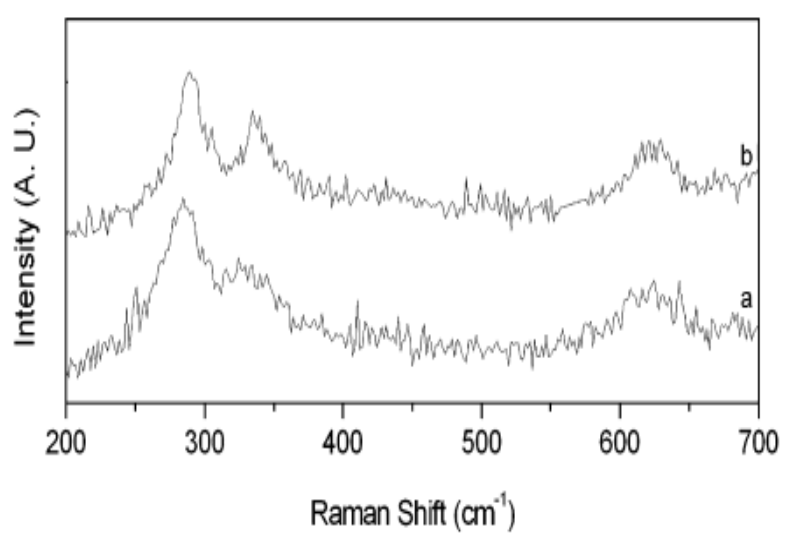

Figure 3. Raman spectra of $\mathrm{CuO}$ samples illustrated in Figs. 1 and $2[4]$

\section{Conclusion}

Well-dispersed $\mathrm{CuO}$ nanocrystals have been synthesized in aqueous solution via a simple hydrothermal procedure. Their shapes can be easily controlled. The shapes of $\mathrm{CuO}$ nanocrystals change as adding temperature of $\mathrm{NaOH}$ change without any additional surfactants. The adding temperatures of $\mathrm{NaOH}$ are critical parameters to the shape-guiding processes.

\section{References}

Qiaobao Zhang, Kaili Zhang, Daguo Xu, Guangcheng Yang, Hui Huang, Fude Nie, Chenmin Liu, Shihe Yang, Progress in Materials Science 60, 208-337 (2014)

2. Xue Wang, Chenguo Hu, Hong Liu, Guojun Du, Xiaoshan He, Yi Xi, Sensors and Actuators B 144, 220-225 (2010)

3. L. Manna, E.C. Scher, A.P. Alivisatos, J. Am. Chem. Soc. 122, 12700 (2000)

4. Junwu Zhu, Huiping Bi, Yanping Wang, Xin Wang, Xujie Yang, Lude Lu, Materials Chemistry and Physics 109, 34-38 (2008)

5. L.S. Birks, H. Friedman, J. Appl. Phys. 17, 687 (1946)

6. Junwu Zhu, Dan Li, Haiqun Chen, Xujie Yang, Lude Lu, Xin Wang, Materials Letters 58, 3324- 3327 (2004)

7. A. Tanaka, S. Onari, T. Arai, Phys. Rev. B 45, 6587 (1992)

8. A. Tanaka, S. Onari, T. Arai, Phys. Rev. B 47, 1237 (1993)

9. Z. Wang, V. Pischedda, S.K. Saxena, P. Lazor, Solid State Commun. 121, 275 (2002).

10. J.F. Xu, W. Ji, Z.X. Shen, S.H. Tang, X.R. Ye, D.Z. Jia, X.Q. Xin, J. Solid State Chem. 147, 516 (1999) 